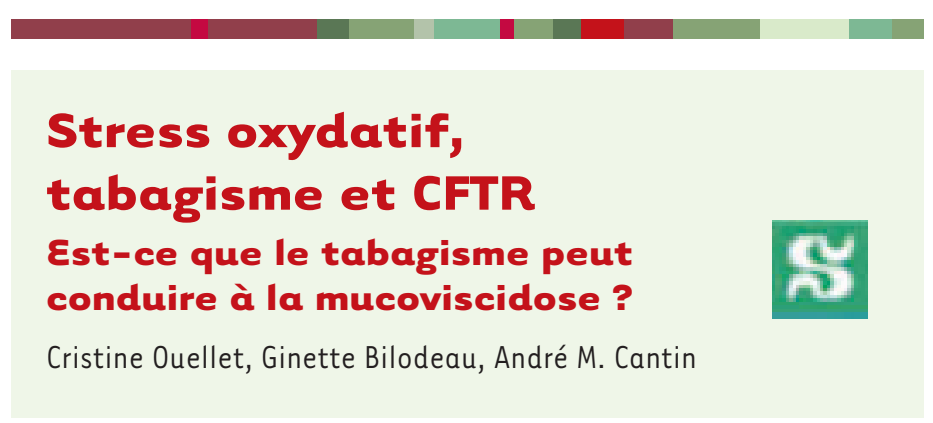

$>$ L'épithélium recouvrant les muqueuses est particulièrement exposé aux agressions. L'arbre broncho-pulmonaire constitue un exemple particulièrement évocateur. Chaque jour, nous respirons plus de 8000 litres d'air dont le contenu en oxygène, un puissant oxydant, est de $21 \%$. L'épithélium broncho-pulmonaire est également exposé aux polluants (dioxyde d'azote, ozone, dioxyde de soufre) d'une planète de plus en plus urbanisée et, comme si ce n'était pas suffisant, le tiers de la population adulte mondiale aspire quotidiennement et de façon volontaire une charge massive d'oxydants provenant de la pyrolyse du tabac. Chaque gramme de goudron provenant d'une cigarette contient $10^{17}$ radicaux libres, plus de 3000 composés aromatiques et de nombreux produits générateurs d'oxydants [1]. La phase gazeuse de la fumée contient $10^{15}$ radicaux par bouffée ainsi que de nombreux oxydes d'azote.

L'épithélium respiratoire forme une vaste frontière qui doit être protégée contre les radicaux libres. Cet épithélium est riche en protéines transmembranaires ABC (ATPbinding cassette proteins), une famille de protéines comprenant 48 membres [2]. Plusieurs de ces protéines effectuent le transport transmembranaire de molécules endogènes et des agents xénobiotiques liés au glutathion, un antioxydant naturel dont la concentration cellulaire est de 1 à $10 \mathrm{mM}$ selon les tissus et de $2 \mathrm{mM}$ dans le poumon. La protéine CFTR (cystic fibrosis transmembrane conductance regulator) est le seul membre de la famille des protéines $A B C$ à être doté de la fonction d'un canal ionique perméable aux anions plutôt que de celle d'un transporteur actif [3]. Nos travaux récents suggèrent que la protéine CFTR pourrait, tout comme d'autres protéines $A B C$, jouer un rôle protecteur contre les radicaux libres car elle est fortement modulée par le stress oxydatif [4].

Le canal CFTR permet le déplacement transmembranaire des anions chlorures, $\mathrm{du}$ bicarbonate, et vraisemblablement $\mathrm{du}$ glutathion, à la surface apicale des cellules épithéliales [5]. La protéine CFTR est produite chez plus de $90 \%$ des sujets porteurs de mucoviscidose, malheureusement elle est défectueuse. La déficience sévère en CFTR qui affecte de manière caractéristique les personnes atteintes de mucoviscidose, entraîne chez elles des infections broncho-pulmonaires répétées. Bien que les manifestations de cette déficience soient connues, les fonctions physiologiques du CFTR restent moins bien définies. Le CFTR joue un rôle déterminant dans I'hydratation du liquide de la surface épithéliale. Lorsque sa fonction est supprimée, non seulement les ions chlorures ne sont pas sécrétés, ce qui engendre une diminution de la sécrétion d'eau, mais en outre le canal $\mathrm{ENaC}$ (canal sodique épithélial) est activé, ce qui entraîne une absorption excessive de sodium et une déshydratation du liquide de la surface épithéliale [6]. De plus, I'absence de CFTR fonctionnel diminue la sécrétion de bicarbonate et de glutathion dans le liquide de la surface épithéliale. La déshydratation, I'acidification et la diminution du glutathion augmentent la viscosité des mucines, les principales protéines du mucus. Ces mucines offrent une importante barrière protectrice pouvant interagir avec les oxydants [7].
Département de médecine,

Unité de Recherche Pulmonaire,

Faculté de Médecine,

Université de Sherbrooke, 3001,

$12^{\mathrm{e}}$ Avenue Nord, Sherbrooke,

Québec, JIH 5N4 Canada.

Andre.Cantin@USherbrooke.ca

La réponse immédiate des cellules épithéliales, dans les minutes suivant une exposition aux oxydants, consiste à activer la fonction du CFTR [8] et à diminuer les taux de glutathion [9] (Figure 1A). En revanche, lorsque des cellules épithéliales telles que les Calu-3 et T84 sont exposées plusieurs heures à un stress oxydatif produit par le tert-butylhydroquinone ( $t$ $B H Q$ ), elles s'adaptent en augmentant la synthèse de glutathion (Figure 1B). Cette adaptation s'accompagne d'une accentuation de la transcription du gène codant la $\gamma$-glutamylcystéine ligase (GCLC), la principale enzyme engagée dans la synthèse du glutathion, qui atteint un taux maximal après quatre à six heures d'exposition au tBHQ. De façon parallèle, le gène encodant le CFTR voit son expression supprimée [4]. Les conséquences d'une suppression du CFTR sont l'augmentation de la concentration et de la viscosité des mucines à la surface de l'épithélium, ainsi qu'une diminution de l'exportation du glutathion, le principal antioxydant cellulaire.

La fumée de la cigarette ainsi que des extraits solubles de cette fumée créent une réponse adaptative au stress oxydatif semblable à celle associée au tBHQ, c'est-àdire une augmentation du glutathion, une accentuation de la transcription du gène GCLC et une suppression de l'expression et de la fonction du CFTR. La fonction du CFTR se mesure chez l'humain en déterminant les différences de potentiel trans-épithélial à la surface de la muqueuse nasale. Chez les sujets porteurs de mucoviscidose, la différence de potentiel basal est nettement exagérée en raison de l'absorption excessive 
de sodium ; de plus la stimulation du canal CFTR avec de l'isoprotérénol n'induit aucun changement de potentiel trans-épithélial nasal [10]. Chez des sujets sains nonfumeurs, la différence de potentiel est plus faible au repos, mais elle augmente à la suite d'une stimulation à l'isoprotérénol. \&n revanche, nous avons démontré qu'il existe une réponse anormale témoignant d'une déficience fonctionnelle du CFTR chez les

\section{A Aigu}

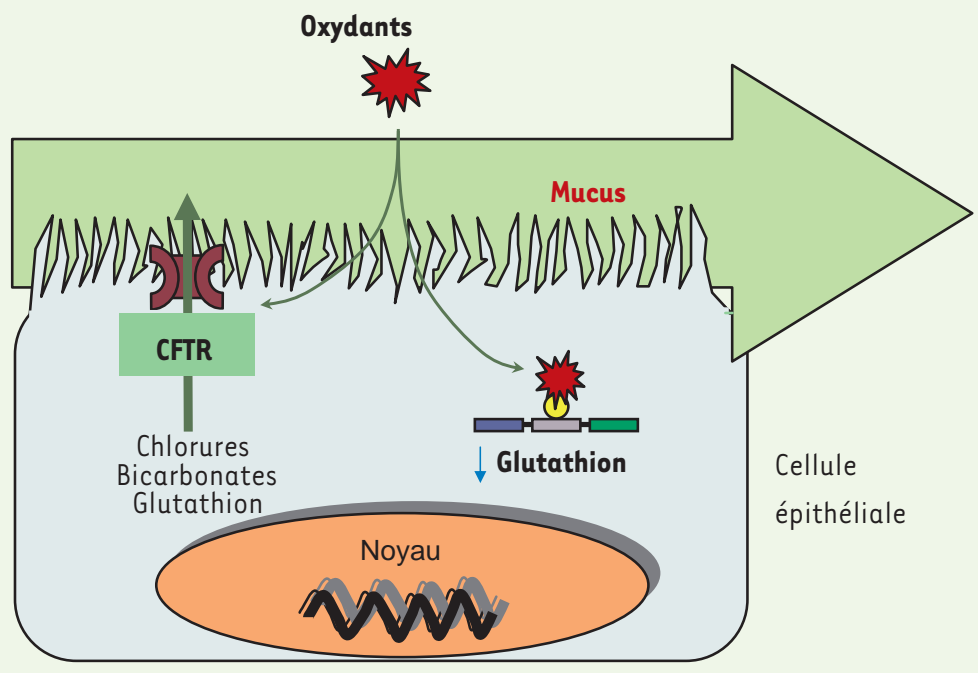

B Chronique

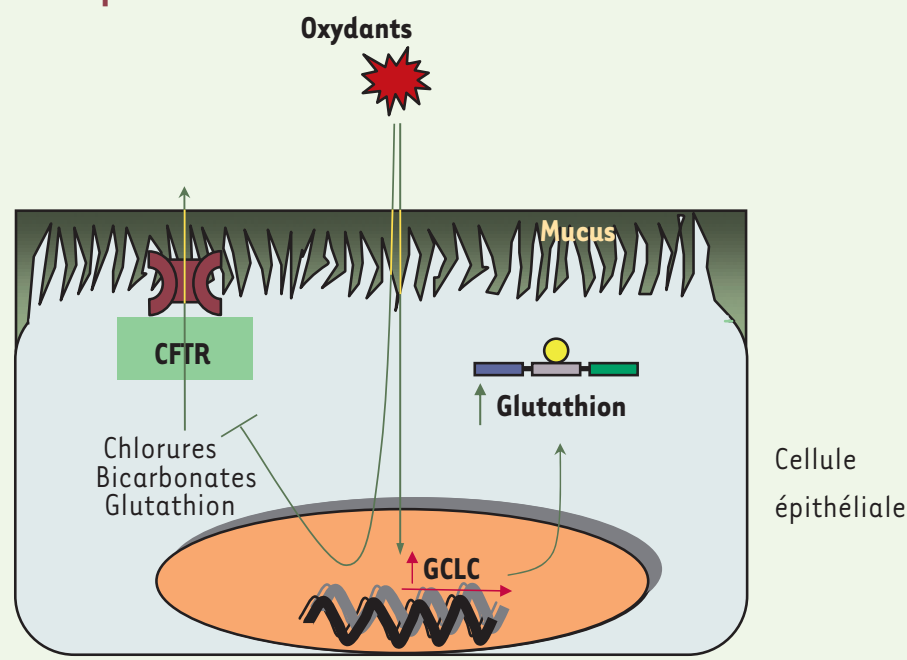

Figure 1. A. Effets aigus du stress oxydatif de l'épithélium sur la fonction du CFTR et les taux cellulaires du glutathion. Dans les minutes suivant une exposition aux oxydants, la cellule épithéliale subit une activation du CFTR et une déplétion du glutathion. Le glutathion réagit avec les oxydants protégeant ainsi la cellule. L'activation du CFTR favorise l'évacuation du mucus pouvant contenir des oxydants et d'autres produits toxiques. B. Effets chroniques d'une exposition de l'épithélium aux oxydants ou à la fumée de cigarettes. Après plusieurs heures d'exposition aux oxydants (ou à la fumée de cigarettes), la transcription du gène GCLC en charge de la synthèse du glutathion est stimulée, cependant que l'expression du CFTR est diminuée. Cette adaptation permet à la cellule de restaurer des taux élevés de glutathion et favorise l'épaississement du mucus protégeant ainsi l'épithélium contre une charge supplémentaire d'oxydants.

fumeurs sains qui ne sont pas atteints de mucoviscidose [11]. Cette déficience chez les fumeurs indique qu'une diminution du CFTR est une réponse physiologique à un stress oxydatif. De plus, les manifestations respiratoires du tabagisme (toux, expectorations, bronchites bactériennes, syndrome obstructif des voies aériennes) sont des signes que l'on retrouve également dans la mucoviscidose. La diminution de l'expression fonctionnelle de la protéine encodée par le gène CFTR en présence d'un stress oxydatif prolongé pourrait contribuer, du moins en partie, aux manifestations cliniques observées chez bon nombre de fumeurs. Ces observations non seulement contribuent à mieux comprendre le rôle physiologique du CFTR, mais encore elles suggèrent que le CFTR détient une part active dans la pathogenèse des bronchopneumopathies obstructives chroniques associées au tabagisme. $\diamond$

Oxidative stress, smoking and CFT: Can cystic fibrosis be acquired?

\section{RÉFÉRENCES}

1. Pryor WA. Biological effects of cigarette smoke, wood smoke, and the smoke from plastics: the use of electron spin resonance. Free Radic Biol Med 1992 ; $13: 659-76$.

2. Van der Deen M, De Vries \&G, Timens W, et al. ATPbinding cassette $(A B C)$ transporters in normal and pathological lung. Respir Res $2005 ; 6: 59$.

3. Riordan JR, Rommens JM, Kerem B, et al. Identification of the cystic fibrosis gene : cloning and characterization of complementary DNA. Science $1989 ; 245$ : 1066-73, 1437.

4. Cantin AM, Bilodeau G, Ouellet C, et al. Oxidant stress suppresses CFTR expression. Am J Physiol Cell Physiol $2006 ; 290$ : C262-70.

5. Linsdell P, Hanrahan JW. Glutathione permeability of CFTR. Am J Physiol 1998 ; 275 : C323-6.

6. Boucher RC. An overview of the pathogenesis of cystic fibrosis lung disease. Adv Drug Deliv Rev 2002; 54 : 1359-71.

7. Perez-Vilar J, Boucher RC. Reevaluating gel-forming mucins' roles in cystic fibrosis lung disease. Free Radic Biol Med 2004 ; 37 : 1564-77.

8. Cowley $\varepsilon A$, Linsdell P. Oxidant stress stimulates anion secretion from the human airway epithelial cell line Calu-3 : implications for cystic fibrosis lung disease. J Physiol 2002 ; 543 : 201-9.

9. Iles KE, Liu RM. Mechanisms of glutamate cysteine ligase (GCL) induction by 4-hydroxynonenal. Free Radic Biol Med 2005 ; 38 : 547-56.

10. Cantin AM, Bilodeau G, Ouellet $C$, et al. Oxidant stress suppresses CFTR expression. Am J Physiol Cell Physiol $2006 ; 290$ : C262-70.

11. Cantin AM, Hanrahan JW, Bilodeau G, et al. Cystic fibrosis transmembrane conductance regulator function is suppressed in cigarette smokers. Am J Respir Crit Care Med 2006 ; 173 : 1139-44. 